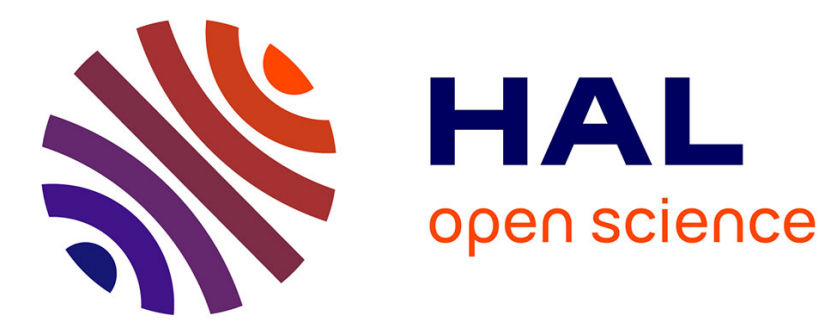

\title{
Anatomy, Evolution of Human
}

Aurélien Mounier

\section{To cite this version:}

Aurélien Mounier. Anatomy, Evolution of Human. The International Encyclopedia of Anthropology, Wiley, 2019, 9781118924396. 10.1002/9781118924396.wbiea1765 . hal-02407580

\section{HAL Id: hal-02407580 \\ https://hal.science/hal-02407580}

Submitted on 12 Dec 2019

HAL is a multi-disciplinary open access archive for the deposit and dissemination of scientific research documents, whether they are published or not. The documents may come from teaching and research institutions in France or abroad, or from public or private research centers.
L'archive ouverte pluridisciplinaire HAL, est destinée au dépôt et à la diffusion de documents scientifiques de niveau recherche, publiés ou non, émanant des établissements d'enseignement et de recherche français ou étrangers, des laboratoires publics ou privés. 


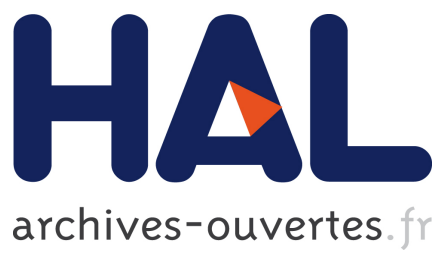

\section{Anatomy, Evolution of Human}

Mounier Aurélien

\section{To cite this version:}

Mounier Aurélien. Anatomy, Evolution of Human. John Wiley \& Sons, Ltd, 2019, 10.1002/9781118924396.wbiea1765 . hal-02407580

\section{HAL Id: hal-02407580 \\ https://hal.archives-ouvertes.fr/hal-02407580}

Submitted on 12 Dec 2019

HAL is a multi-disciplinary open access archive for the deposit and dissemination of scientific research documents, whether they are published or not. The documents may come from teaching and research institutions in France or abroad, or from public or private research centers.
L'archive ouverte pluridisciplinaire HAL, est destinée au dépôt et à la diffusion de documents scientifiques de niveau recherche, publiés ou non, émanant des établissements d'enseignement et de recherche français ou étrangers, des laboratoires publics ou privés. 


\title{
Anatomy, Evolution of Human
}

\author{
AURÉLIEN MOUNIER \\ Musée de I'Homme, France, and University of Cambridge, United Kingdom
}

The evolutionary history of our species, Homo sapiens, is the subject of numerous studies in various subfields of physical anthropology, ranging from primatology and behavioral studies of our closest living relatives to genomics studies, including ancient DNA study of past populations. However, the greatest part of the data available to researchers remains the rather scarce and incomplete fossil record. Its peculiar nature-usually only hard tissues, teeth, and bones survive the fossilization process-has led to the predominance of skeletal anatomical studies in human evolution. In paleoanthropology, the study of skeletal remains follows two principal aims: first, understanding the phylogenetic relationships between humans and their closest extinct relatives and, second, uncovering the way of life of extinct hominin species (their diet and locomotion) in order to infer hypotheses about their ecological niche.

Therefore, paleoanthropology relies predominantly on comparative skeletal anatomy techniques conducted on humans and their closest relatives, both extinct and living. In order to understand the skeletal evidence in the fossil record, one needs a model to interpret it. This model is commonly drawn from the study of the closest living relative of Homo sapiens: the great apes, members of the subfamily Homininae, which is composed of three tribes: Gorillini, Panini, and finally Hominini. The Gorillini and Panini tribes together contain the four species of great apes: western gorillas (Gorilla gorilla), eastern gorillas (Gorilla beringei), chimpanzees (Pan troglodytes), and bonobos (pan paniscus) (Roskov et al. 2015). The Hominini is composed of a unique living species (Homo sapiens) and their closest extinct relatives, classified into many varied and debated genera, from the possible first Hominini (such as Sahelanthropus, Orrorin, and Ardipithecus) to the famous australopithecines (often divided between gracile Australopithecus and robust Paranthropus) and fi nally to the genus Homo, in which are classifi ed living humans along with many debated ancestral species such as Homo habilis, Homo rudolfensis, Homo ergaster, Homo erectus, and Homo heidelbergensis as well as sister species such as Homo neanderthalensis. This entry focuses on the skeletal anatomy of living humans (Homo sapiens) within an evolutionary framework, using the Homininae as a contrast with our own anatomy.

\section{Anatomy in the framework of human evolution}

Human evolutionary studies usually rely on fossilized remains, making skeletal anatomy one of the major domains of paleoanthropologists for understanding the history of our species. Such a focus on anatomy has led to the hyperspecialization of 

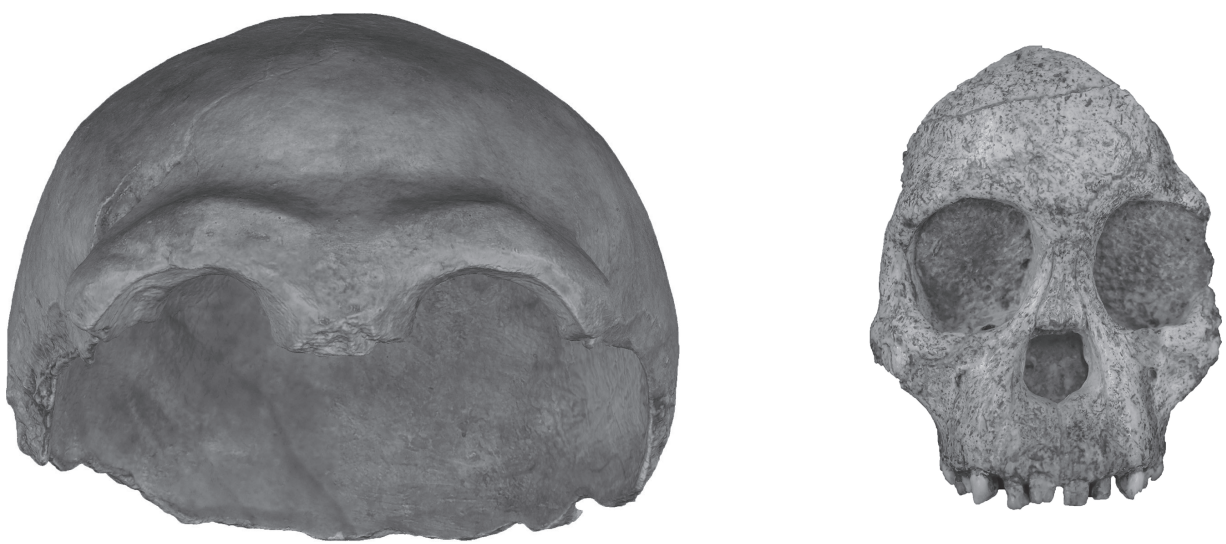

Figure 1 Left: norma facialis of the first Homo neanderthalensis, found in 1856 (cast of Neanderthal 1 from Feldhofer Grotto, Germany). Right: the first australopithecine, found in 1925 (original of Taung 1 from Taung, South Africa).

researchers and to the emergence of two subfields: cranial anatomy and postcranial anatomy. This artificial separation of skeletal elements comes directly from the history of the discipline. For many years, fossilized skulls were the major focus of paleoanthropologists, based on spectacular and important discoveries relating to cranial elements. For instance, the first fossil of a Neanderthal (discovered in 1856 in Feldhofer Grotto, Germany) and the first fossil of an australopithecine (the Taung Child, discovered in South Africa in 1925) were almost completely composed of cranial elements (Figure 1). In the case of the first Neanderthal, the discovery was made by miners, so most of the skeleton was lost; however, some parts of the skeleton were retrieved, notably parts of the limbs. The anatomy of the head in these fossils led researchers to question the established hypotheses on the origin of humans. In contrast, the postcranial evidence in human evolution is less spectacular and was overlooked between the dawn of paleoanthropology (at the end of the nineteenth century) to the beginning of the 1950s.

Another reason for this artificial separation may be found in the fact that the skull, and especially the neurocranium (the cranium without the face), carries a clearer phylogenetic signal than postcranial bones, which makes it more appropriate when trying to understand the phylogenetic relationships that link past populations together. Many studies (e.g., Howells 1989; Lahr 1996) have found differences within extant human populations by looking at the cranial anatomy alone, and many phylogenetic hypotheses regarding the genus Homo come from the same evidence (Mounier, Marchal, and Condemi 2009; Rightmire 1990; Wood 1991). In contrast, the postcranial skeleton is more influenced by climate, diet, and the sociocultural environment than the cranial skeleton, and differences between taxa often reflect differences in niche (Harcourt 2012; Holliday 1997; Ruff 2002).

The skull is composed of 30 bones and the postcranial skeleton of 176 , making a total of 206 bones for the whole skeleton; this number is higher in the newborn at 270, as many bones fuse together during growth. 

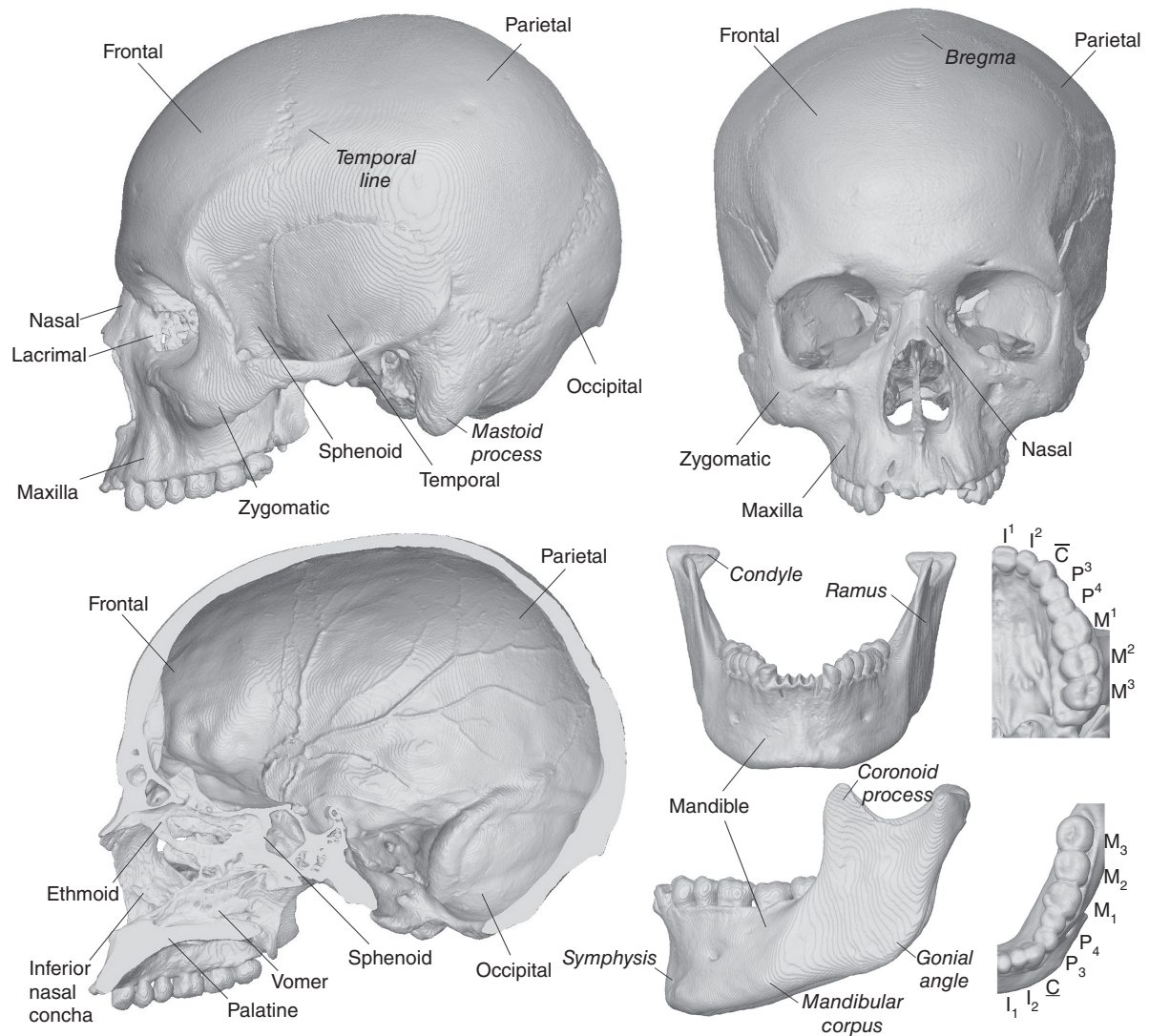

Figure 2 Anatomy of the human skull. From top to bottom and left to right: cranium in norma lateralis, cranium in norma facialis, mid-sagittal section of the cranium, mandible in norma facialis and lateralis, and upper and lower tooth rows. The nonitalic labels refer to bone or tooth names and the italic labels refer to morphological part of a bone.

So $O$ Modified with permission from the Duckworth Collection, University of Cambridge.

\section{Cranial anatomy}

As an anatomical convention, the human skull is generally divided into three parts (Figure 2): the calvarium or neurocranium, composed of eight bones (frontal, parietals left and right, temporal left and right, occipital, sphenoid, and ethmoid) articulated around the brain; the upper face, composed of fourteen bones (zygomatic left and right, maxilla left and right, nasal left and right, lacrimal left and right, inferior nasal conchae left and right, palatines left and right, ethmoid, and vomer), supports the upper tooth row (upper jaw) and provides the basic shape of the face; and the mandible (lower jaw) is a unique bone that supports the lower row of teeth and is attached to the upper face and to the cranium by soft tissues.

In addition to these main cranial bones, there are seven other bones that are usually considered to be cranial bones. Three small bones are found in either middle-ear chambers in the petrous portion of the temporal bone (the auditory ossicles): the malleus, the incus, and the stapes. Their role is to convey sound from the tympanic membrane 
to the inner ear. The hyoid bone is a small horseshoe-shaped bone positioned just above the larynx (approximately at the level of the third cervical vertebra) and plays a role in supporting the tongue. The hyoid is unique in human anatomy as it does not articulate directly with any other bone.

The cranial skeleton also includes the dental anatomy. Each side of each jaw, as in other mammals, contains four types of teeth: from front to back, two incisors, one canine, two premolars, and three molars, making a total of sixteen upper teeth and sixteen lower teeth. The human dental formula is 2.1.2.3/2.1.2.3 (i.e., number per type of teeth from front to back, upper dental row/lower dental row), and is shared with the apes and some other primates. The earliest mammals had a different dental formula: three incisors instead of two, and four premolars instead of two. By convention, the incisors are called I1, I2, and I3; the canine C; the premolars P1, P2, P3, and P4; and the molars M1, M2, and M3. When applicable, superscripts indicate upper teeth (e.g., $\mathrm{I}^{1}$ ) and subscripts indicate lower teeth (e.g., $\mathrm{P}_{3}$ ). Like most living mammals, the Hominidae have lost teeth through evolution. The remaining teeth have kept the ancestral numbering depending on which tooth was lost; the human teeth are named as follows: I1, I2, C, P3, P4, M1, M2, and M3 (Figure 2). Teeth are important in the study of human evolution as they are the part of the skeleton that is best preserved in the fossil record.

\section{Postcranial anatomy}

The postcranial skeleton is divided into two regions: the axial skeleton consists of the skull (which is generally treated separately; see previous section), the vertebral column, and the thorax; the appendicular skeleton consists of the limbs and their connections to the axial skeleton (the shoulders and the hips).

The vertebral column, also called the spine, is the main region of the axial skeleton. It ensures the connection of the brain to the rest of the body thanks to the spinal cord, which runs inside the vertebral canal. The spine can be divided into five parts: seven cervical vertebrae articulate with the skull through the atlas, the first cervical vertebra (C1); twelve thoracic vertebrae connect with the ribs (i.e., the thoracic bones forming the rib or thoracic cage); five lumbar vertebrae articulate with the fourth part of the spine (the sacrum, which consists of five fused sacral vertebrae), which is prolonged by the coccyx (i.e., the vestigial tail). The number of vertebrae in each part may vary slightly between individuals. With the exception of the coccyx, each of those regions has a curvature that is characteristic of the upright posture of bipeds (Figure 3; Figure 4). The thorax provides support and protection for numerous organs, including the lungs and the heart. It is composed of twenty-four ribs (twelve on each side) that articulate with the thoracic vertebra posteriorly and anteriorly with the sternum. The sternum is also articulated with the clavicle.

On each side of the body, the appendicular skeleton is formed in the upper part of the body by the shoulders (composed of the scapula and the clavicle) and by the arms and hands. The arms have three long bones: the humerus, which articulates proximally with the scapula and distally forms the elbow with the radius (laterally) and ulna (medially), which in turn compose the lower arm and which articulate with the wrist. The wrist is formed of eight smaller bones articulated together and with the proximal hand 


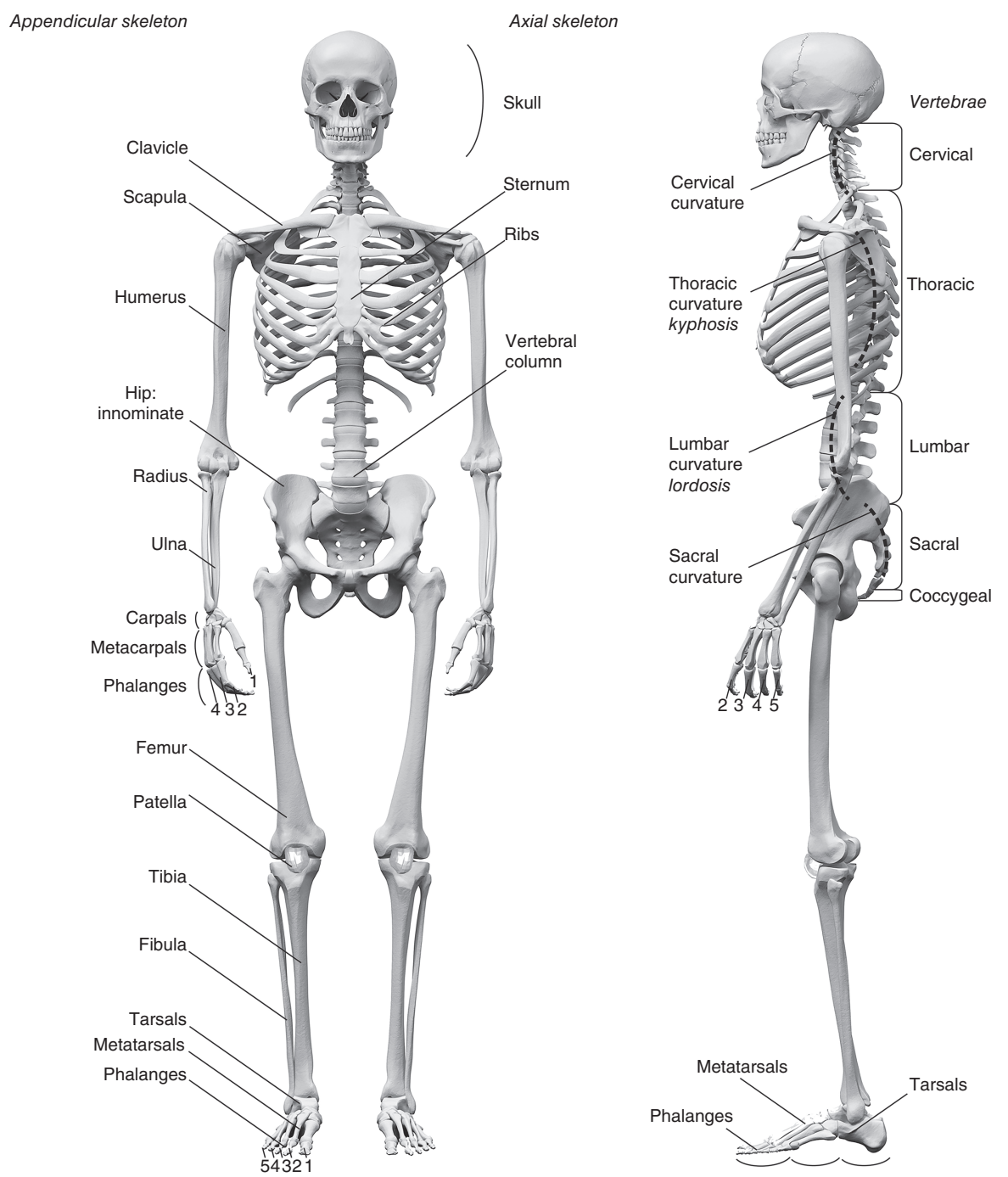

Figure 3 Human postcranial anatomy. The norma facialis view (left) of the human skeleton shows the appendicular (left set of labels) and axial (right set of labels) skeletons. The norma lateralis view (right) highlights the morphology of the vertebral column and the foot morphology. Sourc Dedified with permission from Leocalvett / Dreamstime.

bones, which are the five metacarpals (one for each finger), which are prolonged by three phalanges, with the exception of the thumb (digit one), which has only two phalanges. In the lower part of the body, the hips are made of the innominate articulated with the sacrum medially and with the leg laterally. Similar to the upper part of the body, the leg is composed of three long bones: the femur articulates proximally with the innominate and distally with the fibula (medially) and the tibia (laterally), forming the knee, with an additional bone, the patella, articulating with the femur and the tibia. Both the tibia and the fibula articulate with the seven ankle bones, which in turn connect to the proximal foot bones, which are the five metatarsals (which are prolonged by the toe bones) 

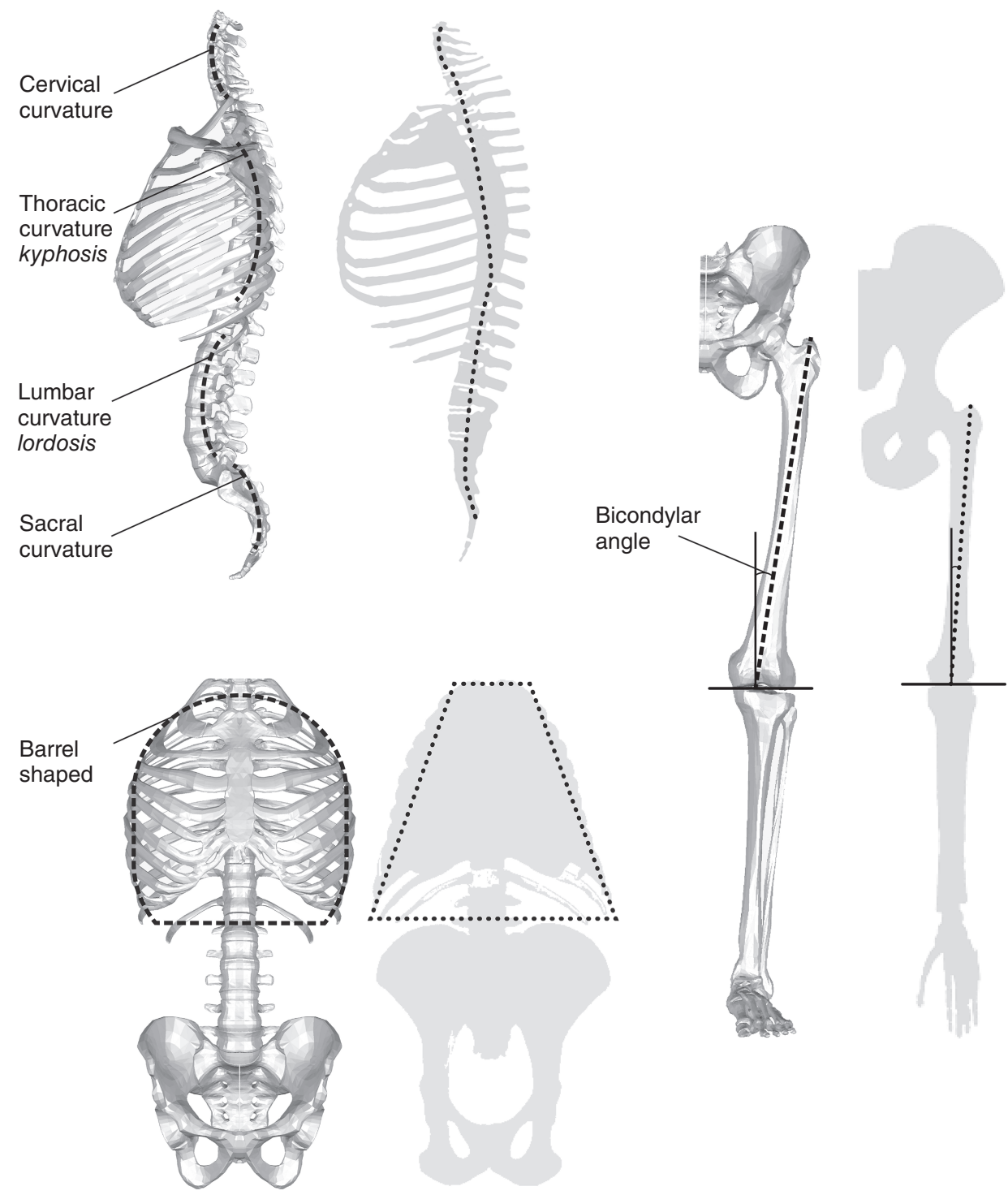

Figure 4 Human postcranial skeleton showing the main adaptations to bipedalism compared with chimpanzee anatomy (shadowed). Top left: norma lateralis of the human vertebral column. Bottom left: vertebral column and rib cage. Right: norma facialis of the pelvis and of a modern human leg. Source: $\sigma$ fied with permission from TurboSquid.

plus three phalanges for each toe with the exception of the hallux (digit one), which has only two phalanges (Figure 3).

\section{Bipedalism: A specialized body}

One of the major morphological characteristics of the subtribe Hominini is the specialization for bipedalism. The human skeleton is especially well designed for this 
mode of locomotion, and humans have a number of anatomical specializations toward bipedalism. Those characteristics can be observed both on the skull and on the rest of the skeleton.

On the cranium, one of the main features linked to bipedalism is the position of the foramen magnum. This large orifice runs through the inferior part of the occipital bone and connects the cranial cavity with the vertebral canal, where the spinal cord, the vertebral arteries, and the accessory nerves go. In bipedal humans, the foramen magnum is positioned below the skull, allowing the cranium to rest atop of the vertebral column; in contrast, it is positioned toward the back of the skull in more quadrupedal primates (including the great apes), preventing a full upright bipedal stance. In other Homo species, including Homo erectus sensu lato, Homo heidelbergensis sensu lato, and Homo neanderthalensis, the foramen magnum is positioned in the same way as in modern humans. In australopithecines, whose bipedalism is slightly different from that of modern humans, the foramen magnum is anteriorly positioned as in Homo but has a different inclination (Kimbel and Rak 2010; Figure 5). Associated with this morphology are the shape and position of the nuchal crests. In humans the insertion of the nuchal muscles (i.e., longissimus capitis and semispinalis capitis) is not strongly marked and is positioned well below the skull, while in great apes there is a strong nuchal crest located superiorly on the skull at the suture of the occipital and the parietals. Within the genus Homo, this muscle insertion is generally more developed than in modern humans and forms a posteriorly located occipital torus in most Homo species (i.e., Homo erectus sensu lato, Homo heidelbergensis, and Homo neanderthalensis). This is also the case in australopithecines, which tend to show a very strongly developed occipital torus (Figure 5).

The postcranial skeleton bears most of the specializations toward bipedalism. In the axial skeleton, the vertebral column, which enters vertically from the center of the skull, shows four distinctive curvatures that play a role in maintaining balance when walking upright. Only the thoracic (kyphosis) and sacral curvatures are present from birth (primary curvatures); two secondary curvatures form later: the cervical curvature at about three months of age and the lumbar (lordosis) curvature when the child begins to walk. In great apes, only chimpanzees have a unique cervical curvature. There is strong evidence (Ward 2013) showing that the australopithecines (both robust and gracile) had developed spinal curvatures, and the lumbar curvature has been identified in numerous fossils. The four curvatures are present in later Homo species and in Homo erectus (Walker and Leakey 1993), showing a perfect adaptation to bipedalism. Additionally, the rib cage reflects adaptation to locomotion. Humans have a barrel-shaped thoracic cage, narrow at the top and the bottom and widened in its medial part, which helps to centralize the body's center of gravity, contrary to the more conic-shaped (narrow at the top and wide at the bottom) thoracic cage of the great apes (Figure 4). Both early Homo and australopithecines show a thoracic cage that is similar to that of humans, even though it is not completely clear in the case of australopithecines, which were first described as having a conic-shaped rib cage (Walker and Leakey 1993). Homo neanderthalensis is believed to have had a barrel-shaped thoracic cage, which could be due to adaptation to a cold environment. 

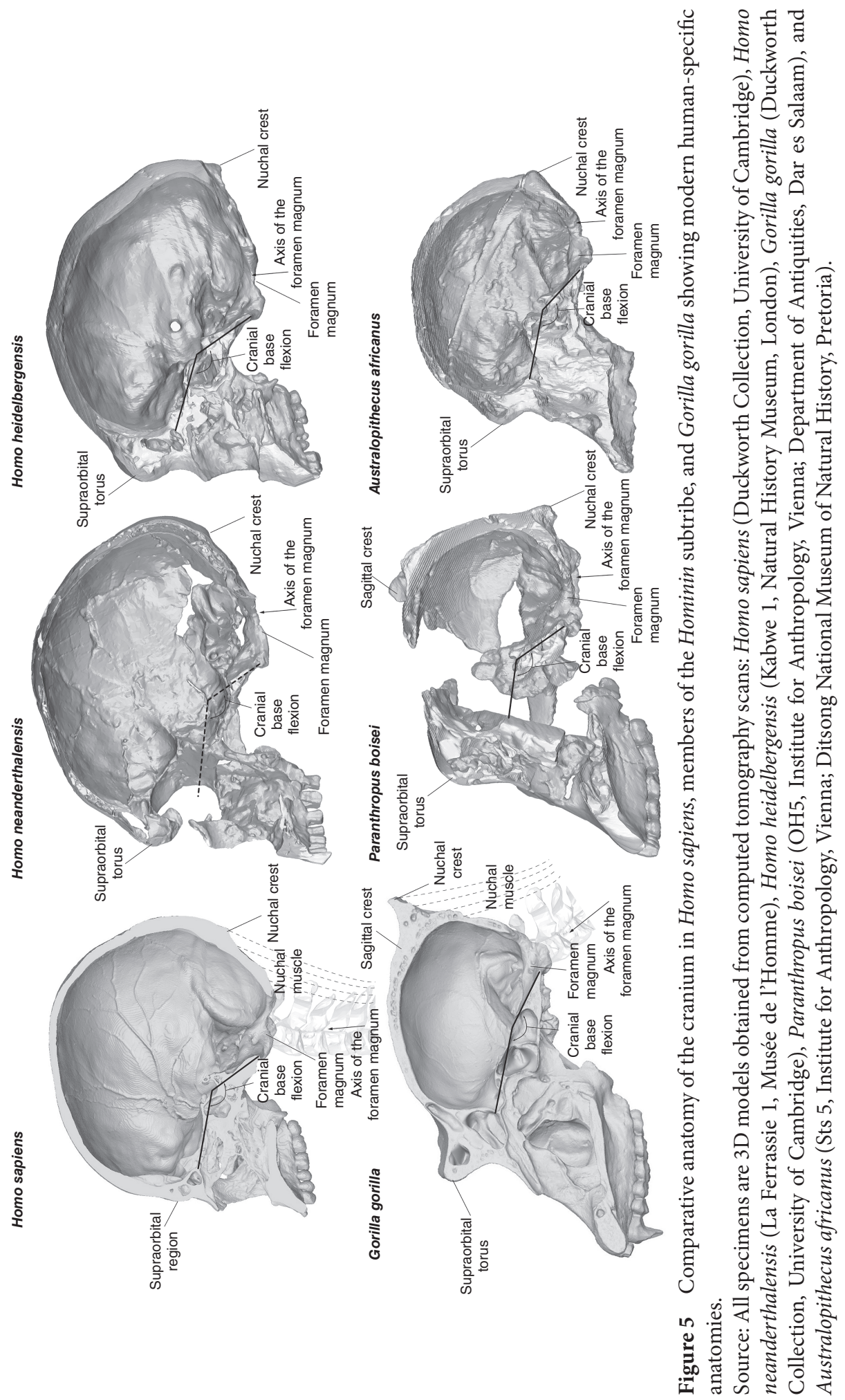
The human appendicular skeleton has much shorter upper limbs than lower limbs; this contrasts with the great apes, where the upper limbs are longer than the lower limbs, illustrating their particular quadrupedal, partly arboreal, knuckle-walking locomotion. In australopithecines, the relative proportion of the lower and upper limbs is somewhat intermediary, with relatively longer arms than in humans, depicting their enhanced abilities for arboreal locomotion compared to humans. Within the genus Homo and in Homo erectus, those proportions are humanlike.

In the upper part of the axial skeleton, the human short arms articulate with the scapula and the clavicle. The scapula is posteriorly positioned and the glenoid cavity (the socket that articulates with the humerus) is laterally oriented, in contrast to great apes, whose scapula is positioned more laterally with the glenoid cavity more superiorly oriented. Ape anatomy gives more flexibility to the upper limbs, while human morphology may favor the use of the upper limbs in an anterior position, hence enhancing the ability to manipulate objects. Most hominins have a humanlike morphology, with the exception of the australopithecines, who may have retained a somewhat more laterally positioned shoulder. Additionally, the human hand shows a major specialization with a fully opposable thumb (digit one; Figure 3), allowing two prehensile grips: the precision and the power grips (Napier 1956). But we lack any specialization toward arboreal locomotion as seen in great apes and australopithecines (i.e., the metacarpals and phalanges of digits two to five are longer and curved). Most of the Hominini belonging to the genus Homo show a similar humanlike condition, indicating their absence of specialization for arboreal behavior.

In the lower part of the appendicular skeleton, one of the main features showing the adaptation to locomotion is the human pelvis, which is supero-inferiorly shortened and latero-medially widened, with the ilium (the thin blade-like portion of the innominate superior to the hip socket) flaring laterally overlooking the leg (Figure 4). The human pelvis forms a large pelvic cavity, allowing the passage of newborns with large heads. In great apes, the hips are supero-inferiorly lengthened and latero-medially shortened. The pelvis of the first bipeds, the australopithecines, is not intermediate in shape: it is broader latero-medially than the human one. This morphology reflects a perfect bipedalism, which is nevertheless different from that of humans. Such a specialization of the hips is accompanied by a different lower-limb anatomy. Specifically, the human femur has a long femoral neck connecting it to the innominate, and is strongly angled (8-11 degrees) compared to vertical orientation. In great apes, the femoral bicondylar angle is almost absent (1-2 degrees) while the australopithecines show an extreme pattern, with 12-15 degrees (Figure 4 ). The ankle and foot are also highly specialized in humans. First, the ankle joint is nearly perpendicular to the long axis of the tibia, while in great apes it is angled (tibiotalar angle: 20-35 degrees); in addition, the tarsals and metatarsals have a different morphology, as exemplified by the development of the human calcaneus (heel), which is adapted to a heel-striking walk and by the presence of longitudinal arches on the inferior part of the foot; finally, the human hallux is aligned with the other digits (laterally rotated and adducted) whereas it is opposable in great apes, offering (with curved phalanges) grasping abilities that are completely absent in humans. 


\section{A unique cranial shape, encephalization, and diet}

Primates in general have relatively large brains in relation to their body size. Encephalization is the relative measure of brain size compared to an expected value for a typical animal of the same body size. In primates this has been linked to various factors, such as intelligence, diet, and the use of binocular vision. However, if relatively large brains are a shared common feature in primates, the degree of encephalization observed in modern humans sets them apart from both the other primates and the other members of the subtribe Hominini, which share a specialization toward bipedalism. Especially, it separates modern humans from the australopithecines, which are habitual bipeds but lack the extreme encephalization observed among the members of the genus Homo.

Modern human cranial capacity, in current populations, is highly variable, ranging from 1,000 to $1,700 \mathrm{~cm}^{3}$, but the average capacity is around $1,400 \mathrm{~cm}^{3}$. This is much bigger than in other great apes, whose cranial capacity is generally around $400 \mathrm{~cm}^{3}$ (respectively for chimpanzees, gorillas, and orangutans: $393 \mathrm{~cm}^{3}, 465 \mathrm{~cm}^{3}$, and $418 \mathrm{~cm}^{3}$ ), but smaller than in Homo neanderthalensis (around $1,500 \mathrm{~cm}^{3}$ ). Relative to body size (or mass), modern humans have the largest brain of all Hominini. The skull is a highly integrated anatomical structure, and covariation between the calvarium (i.e., neurocranium), the face, and the mandible has been well described (Lieberman 2011). To accommodate such a massive brain, many anatomical characteristics of the various regions of the skull are specialized.

The first striking feature is the globular (i.e., spherical) shape of the cranial vault (neurocranium), which is unique to the Homo genus and more particularly to Homo sapiens. In addition to providing more space for the brain, biomechanical characteristics of the spherical shape of the vault-associated with a thicker cranial vault in critical locations (at the bregma: the suture between the frontal and parietal bones, and at the back of the head on the occipital; Figure 2; Figure 5) -may help to protect the brain from localized impacts and prevent fractures. The reduction of the cranial base angle is associated with encephalization: modern humans have a highly flexed basicranium whose evolution was probably driven by the increase of brain size without any increase in basicranium length. The cranial base angle is approximately 157 degrees in gorillas, whereas it is around 142 degrees in australopithecines (Australopithecus afarensis) and only 137 degrees in modern humans. A consequence of a flexed basicranium in modern humans is the downward rotation of the face, which is located underneath the anterior-most part of the brain case. In a comparative perspective and also highly correlated to the increase in cranial capacity, the relative proportions of the face and the calvarium are also remarkable (Figure 3 ). In great apes, the face occupies as much space in the skull as the calvarium, while in modern humans the calvarium is much larger than the face. The development of a larger brain throughout human evolution was accompanied by a reduction in facial proportion and in facial projection. The human face is called "orthognathic," as it does not project anteriorly and is relatively flat, while the great apes and the australopithecines have "prognathic" faces, which project anteriorly. Most hominins, including numerous Homo species, have prognathic faces, which are, however, much reduced compared to those of great apes and of australopithecines, but 
only modern humans have faces that are properly orthognathic and located underneath the brain.

Another aspect that impacted the evolution of the human skull is related to diet. Humans eat highly processed and transformed (i.e., cooked) food, which allows them to eat very hard types of food without having a specialized morphology. In contrast, great apes spend a lot of their time eating and chewing hard types of food. This can be seen in the shape of their skull and primarily in the strongly marked insertions of the main chewing muscles (i.e., temporalis, masseter, and medial and lateral pterygoid). For instance, the temporalis muscle, which runs from the parietal and frontal bones to the coronoid process of the mandible (Figure 2), leaves faint marks at mid-height on the parietal and frontal bones of the human skull (the temporal line), while in great apes and in robust australopithecines the right and left muscles join in the midline of the skull and the insertion forms a strongly developed bony crest (sagittal crest; see Figure 5). Homo species completely lack this superstructure, which is reduced in Homo erectus to a slight increase in cranial thickness at the level of the bregma and to temporal lines that may form a swelling on the frontal and parietal bones. Similarly, the masseter muscle, which runs from the zygomatic bone to the gonial angle of the mandible, may or may not leave a mark on the human mandible, whereas it does in great apes and in australopithecines, which often show extroverted gonial angles. This particular feature may be observed within the genus Homo, especially in Homo erectus fossils. More generally, the shape of the skull may be affected by the type of food processed. For instance, the human mandible has a rather short antero-posterior ramus length and a relatively short supero-inferior mandibular corpus length (Figure 2). Both great apes and australopithecines have wider rami and taller mandibular corpora. This is also the case for most premodern Homo species. However, it is important to keep in mind that the facial architecture is plastic and may reflect local adaptation to diet.

Finally, teeth also reflect shifts in diet, and teeth morphology is commonly used in the identification of fossil specimens. Overall, the human denture is small, especially when compared to that of both great apes and australopithecines. First, humans have small canines, a trait that is mostly related to sexual dimorphism, and consequently lack a diastema (a space between P3 and C), which allows oversized canines to fit into the mouth. Second, humans have relatively small postcanine teeth-premolars and molars (Figure 2). In australopithecines and especially in Paranthropus, the premolars and molars are highly specialized and feature extreme proportions (i.e., megadontia) compared to the front teeth. Within the genus Homo, these features are always absent, and Homo species such as Homo heidelbergensis may have a different skeletal morphology, but the major dental traits are modernlike.

Finally, two additional morphologies of the human skull may or may not be related to diet but are important in the definition of Homo sapiens. The first is the presence of a chin on the anterior surface of the symphysis of the mandible. No other primate, no other Hominini, and no other Homo species possess such a swelling on the mandible, which is formed by bone resorption of the upper alveolar portion of the symphysis, leaving a projecting chin. Many hypotheses regarding the biomechanical properties of the human chin have been made, but none of them is completely convincing in making 


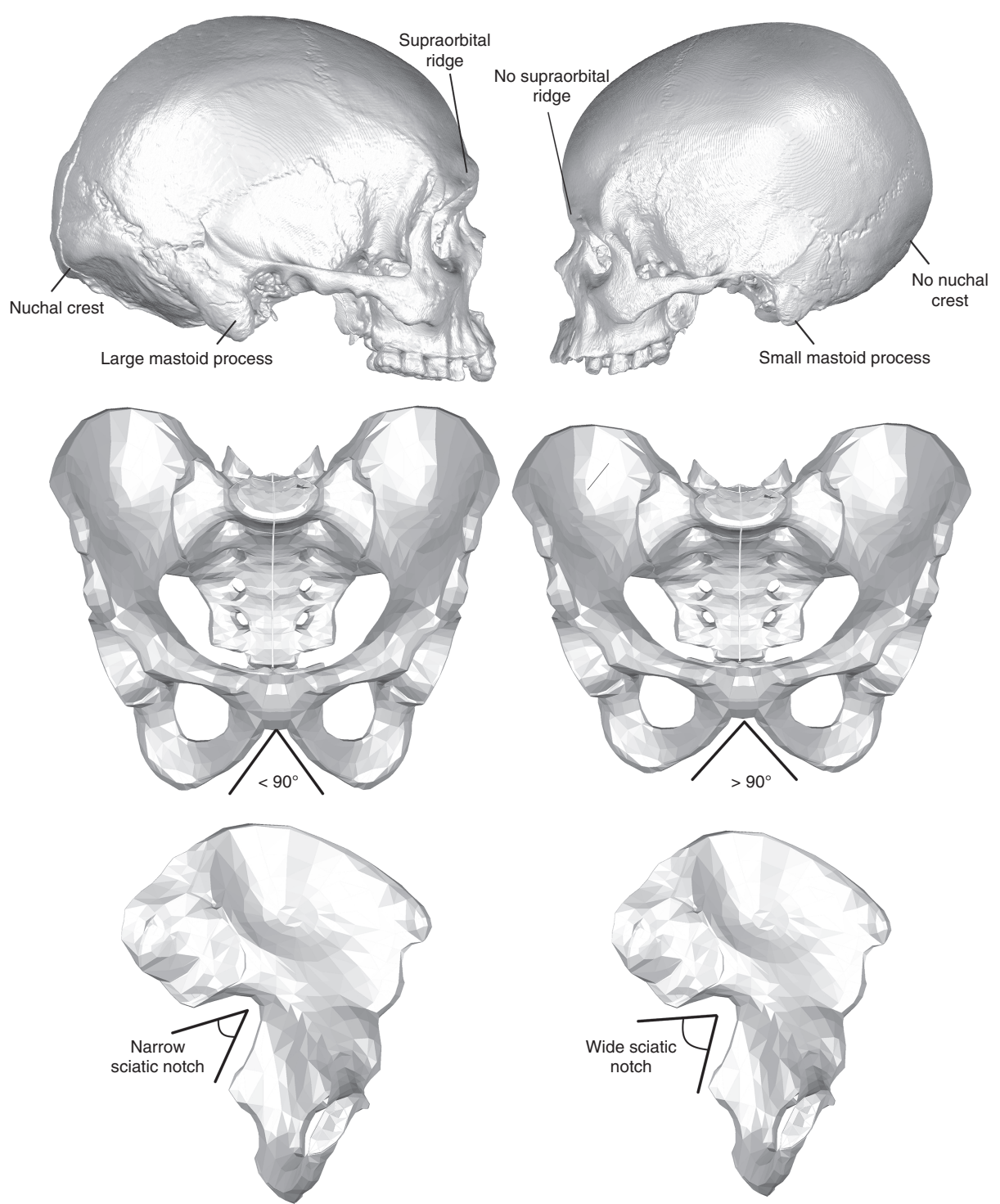

Figure 6 Differences between male (left) and female (right) Homo sapiens on the cranium and on the pelvis. From top to bottom: norma lateralis of a male and female cranium, norma frontalis of male and female pelvises, and norma lateralis of male and female pelvises.

Sourc permission from the Duckworth Collection, University of Camb2; middle and bottom images modified with permission from TurboSquid.

the chin an adaptation to mechanical strain (Lieberman 2011). The other morphology is the absence of a supraorbital torus or supraorbital bar, which is a relatively straight and continuous bony superstructure that extends across the frontal bone and projects anteriorly above the orbits (Figure 5). Both great apes and australopithecines have a strongly marked supraorbital torus. Most Homo species have such a superstructure but modern humans do not. The lack of a supraorbital torus in modern humans is linked to their 
small face and the lack of upper facial projection. However, various studies have pointed out that a supraorbital torus may have implications in strains related to chewing (Picq and Hylander 1992), but this functional inference has not been fully demonstrated.

\section{Sexual dimorphism in humans}

In most Homininae, females and males possess different morphologies, reflecting their sex. For instance, in gorillas, the cranial superstructures (sagittal crest, nuchal crests) and the canines are much more developed in males than in females. Within the Hominini subtribe, such sexual differences tend to reduce over time. Australopithecines seem to have displayed a relatively strong sexual dimorphism, but within the Homo genus the differences have almost become unnoticeable. In modern humans, females are often smaller and generally more gracile, and a few cranial morphological features may differ between male and female. For instance, males may have a supraorbital region developing into a ridge, strong muscle insertions on the occipital (nuchal crests), and a strongly developed mastoid process. Additionally, the frontal bone is often steeper in females and more rounded in males (Figure 6). However, most of those features may vary between and within populations without always being related to sex.

The most important morphological difference between males and females is to be found in the pelvis. One of the functions of the pelvis is linked to parturition. As a result, females have a slightly different pelvis as it has to accommodate the transit of newborns: the cavity of the pelvis as well as the pelvic inlet and outlet are wider in females; the angle of the pubic arch is greater than 90 degrees and the sciatic notch is wider (Figure 6).

The human skeletal anatomy underlines the main aspects of our evolution by showing how our skeleton reflects both our taxonomical classification and our phylogenetic relationships with our closest relatives.

SEE ALSO: Skeletal Aging and Sexing Techniques; Homo: Evolution of the Genus; Anatomy and Climate-Related Variation in Hominins; Modern Humans, Origins of; Hominins, Early; Childbirth and Brain Size, Evolutionary Constraints of; Bipedalism

\section{REFERENCES AND FURTHER READING}

Harcourt, Alexander H. 2012. Human Biogeography. Berkeley: University of California Press. Holliday, Trenton W. 1997. "Postcranial Evidence of Cold Adaptation in European Neandertals." American Journal of Physical Anthropology 104: 245-58.

Howells, William W. 1989. Skull Shapes and the Map: Craniometric Analyses in the Dispersion of Modern Homo. Cambridge, MA: Harvard University Press.

Kimbel, William H., and Yoel Rak. 2010. "The Cranial Base of Australopithecus afarensis: New Insights from the Female Skull." Philosophical Transaction of the Royal Society B: Biological Sciences 365: 3365-76.

Lahr, Marta M. 1996. The Evolution of Modern Human Diversity: A Study of Cranial Variation. Cambridge: Cambridge University Press. 
Lieberman, Daniel E. 2011. The Evolution of the Human Head. Cambridge, MA: Harvard University Press.

Mounier, Aurélien, François Marchal, and Silvana Condemi. 2009. "Is Homo heidelbergensis a Distinct Species? New Insight on the Mauer Mandible." Journal of Human Evolution 56: 219-46.

Napier, J. R. 1956. “The Prehensile Movements of the Human Hand.” Journal of Bone \& Joint Surgery 38-B (4): 902-13.

Picq, Pascal G., and William L. Hylander. 1992. "La signification fonctionnelle du torus sus-orbitaire et la transition Homo erectus / Homo sapiens [The Functional Significance of the Supra-orbital Torus and the Transition Homo erectus / Homo sapiens]." In Cinq millions d'années, l'aventure humaine [Five Million Years, the Human Journey], edited by M. Toussaint, 125-50. Liège, Belgium: ERAUL.

Rightmire, G. Philip. 1990. The Evolution of Homo erectus: Comparative Anatomical Studies of an Extinct Human Species. Cambridge: Cambridge University Press.

Roskov, Y., L. Abucay, T. Orrell, D. Nicolson, T. Kunze, A. Culham, N. Bailly et al. 2015. Species 2000 \& ITIS Catalogue of Life: 2015 Annual Checklist. Accessed June 22, 2017, http://www. catalogueoflife.org/annual-checklist/2015.

Ruff, Christopher. 2002. "Variation in Human Body Size and Shape." Annual Review of Anthropology 31: 211-32.

Walker, Alan C., and Richard E. F. Leakey, eds. 1993. The Nariokotome Homo erectus Skeleton. Berlin: Springer.

Ward, Carol V. 2013. "Postural and Locomotor Adaptations of Australopithecus Species." In The Paleobiology of Australopithecus, edited by Kaye E. Reed, John G. Fleagle, and Richard E. Leakey, 235-46. Dordrecht: Springer.

White, Tim D., Michael T. Black, and Pieter A. Folkens. 2011. Human Osteology. 3rd ed. Amsterdam: Academic Press.

Whitehead, Paul F., William K. Sacco, and S. B. Hochgraf. 2005. A Photographic Atlas for Physical Anthropology. Englewood, CO: Morton.

Wood, Bernard. 1991. Hominid Cranial Remains. Vol. 4 of Koobi Fora Reserach Project. Oxford: Clarendon Press.

Wood, Bernard, ed. 2011. Wiley-Blackwell Encyclopedia of Human Evolution. Chichester, UK: Wiley Blackwell. 
Please note that the abstract and keywords will not be included in the printed book, but are required for the online presentation of this book which will be published on Wiley's own online publishing platform.

If the abstract and keywords are not present below, please take this opportunity to add them now.

The abstract should be a short paragraph of between 50 and 150 words in length and there should be at least 3 keywords.

\section{ABSTRACT}

The study of the evolution of humans relies mostly on data from comparative skeletal anatomy techniques conducted on Hominini remains. This entry describes the skeletal anatomy of modern humans (Homo sapiens) within the framework of human evolution, contrasting modern human specialized cranial and postcranial skeletons with the skeletons of great apes (i.e., gorillas and chimpanzees) and with other species from the Hominini subtribe (i.e., Australopithecus, Paranthropus, and other Homo species).

\section{KEYWORDS}

biological or physical anthropology; evolution; human evolution; paleoanthropology; skeletal anatomy 